\title{
SPECTROSCOPIC BINARIES IN THE ORION NEBULA CLUSTER AND NGC 2264
}

\author{
Marina Kounkel ${ }^{1}$, Lee Hartmann ${ }^{1}$, John J. Tobin ${ }^{2}$, Mario Mateo ${ }^{1}$, John I. Bailey, III $^{1}$, And Meghin Spencer ${ }^{1}$ \\ ${ }^{1}$ Department of Astronomy, University of Michigan, 1085 S. University Street, Ann Arbor, MI 48109, USA \\ ${ }^{2}$ Leiden Observatory, Leiden University, P.O. Box 9513, 2300-RA Leiden, The Netherlands; mkounkel@umich.edu \\ Received 2015 November 16; accepted 2016 February 16; published 2016 April 1
}

\begin{abstract}
We examine the spectroscopic binary population for two massive nearby regions of clustered star formation, the Orion Nebula Cluster (ONC) and NGC 2264, supplementing the data presented by Tobin et al. with more recent observations and more extensive analysis. The inferred multiplicity fraction up to 10 au based on these observations is $5.3 \pm 1.2 \%$ for NGC 2264 and $5.8 \pm 1.1 \%$ for the ONC; these values are consistent with the distribution of binaries in the field in the relevant parameter range. Eight of the multiple systems in the sample have enough epochs to perform an initial fit for the orbital parameters. Two of these sources are double-lined spectroscopic binaries; for them, we determine the mass ratio. Our reanalysis of the distribution of stellar radial velocities toward these clusters presents a significantly better agreement between stellar and gas kinematics than was previously thought.
\end{abstract}

Key words: binaries: spectroscopic - ISM: individual objects (ONC, NGC 2264) - ISM: structure - stars: formation

Supporting material: machine-readable table

\section{INTRODUCTION}

Approximately half of Sun-like stars belong to binary or higher-order multiple systems (Raghavan et al. 2010), and this fraction increases for more massive primaries (e.g., Duquennoy \& Mayor 1991; Kouwenhoven et al. 2007; Rizzuto et al. 2013). Observations of several nearby non-clustered, star-forming regions and associations revealed that they contain a larger fraction of multiple systems compared to the significantly more evolved field stars. On the other hand, high-resolution imaging suggests that some young clusters are deficient in binaries (Duchêne \& Kraus 2013 and references therein). Since most stars are thought to form in dense clusters (Adams 2010), studies of binary frequency as a function of young cluster structure and dynamics can shed light on the processes behind the present day stellar multiplicity.

The Orion Nebula Cloud (ONC) and NGC 2264 are two of the closest regions of clustered star formation $(d \sim 400 \mathrm{pc}$ and $\sim 900 \mathrm{pc}$, ages of $1-2 \mathrm{Myr}$ and $1.5-3 \mathrm{Myr}$, respectively; Hillenbrand 1997; Sung et al. 1997; Menten et al. 2007; Sandstrom et al. 2007; Baxter et al. 2009). While the binarity of nearby stars is relatively well understood, it becomes increasingly more difficult to characterize the full membership of multiple stars in young star-forming regions due to their larger distances. Nonetheless, extensive studies of optical binaries in the ONC have led to interesting findings. Most of the studies concentrated on wider binaries that can be detected through photometric surveys with a smallest separation between two companions of $\sim 60 \mathrm{au}$, set by the diffraction limit of the optics (Köhler et al. 2006; Reipurth et al. 2007). A recent survey of Class I and II stars in the Orion Molecular Clouds by Kounkel et al. (2016) revealed that, contrary to expectations, densely populated regions have a larger fraction of wide multiple systems than diffusely populated ones, highlighting the need to reexamine the environmental dependence on the evolution of multiplicity. Constructing a more well-defined sample of close binary systems that can be obtained through multi-epoch spectroscopic monitoring is an important step in this process.
In addition, identifying spectroscopic binaries and removing them from the sample in kinematic studies of star-forming regions can help refine tests of cluster formation. There is some debate over whether clusters form in a slow process, taking place in clouds initially supported by supersonic turbulence (e.g., Tan et al. 2006; Hennebelle 2012), or whether clusters form rapidly on a free-fall scale due to gravitational collapse (e.g., Elmegreen 2007; Hartmann \& Burkert 2007; Kuznetsova et al. 2015). Simulations have shown that in the case of the former, any subclustering in the initial environment would not change significantly in the cluster evolution; for the latter, any substructure would rapidly dissipate in only a few Myr (e.g., Scally \& Clarke 2002). By examining the cluster dynamics of massive and clustered star-forming regions, it is possible to set important constraints on models that would more effectively distinguish between these two theories.

Tobin et al. (2009, hereafter T09) attempted to identify spectroscopic binaries, which typically have significantly narrower separations, using multi-epoch spectroscopic monitoring of 1613 objects toward the ONC, searching for variability in the radial velocities (RVs) which can be attributed to the presence of a companion. NGC 2264 has not yet been the subject of a systematic binary survey, although Tobin et al. (2015, hereafter T15) did report on multi-epoch spectra toward 695 objects in the direction of this region.

T09 and T15 have previously analyzed the kinematic structure of the ONC and NGC 2264, building on the efforts of Funrész et al. (2006, 2008). They found that in both of these regions, there is general agreement in RV between stars and the gas from which they formed, which suggests that these regions are dynamically young with ages of 1-2 crossing times. Surprisingly, however, a significant fraction of the stars appeared to be blueshifted relative to the gas, and there does not appear to be a significant number of redshifted sources to balance the distribution. T15 showed that the spectra of some of these blueshifted objects found toward NGC 2264 exhibit Li I $6707 \AA$ absorption. This is an indicator of extreme youth, and while this is not a confirmation of the membership of the 
Table 1

Dates and Configurations of the Observations ${ }^{\mathrm{a}}$

\begin{tabular}{|c|c|c|c|c|c|}
\hline $\begin{array}{l}\text { Field } \\
\text { ID }\end{array}$ & $\begin{array}{l}\text { Date } \\
\text { (UT) }\end{array}$ & $\begin{array}{c}\text { R.A. } \\
(\mathrm{J} 2000)\end{array}$ & $\begin{array}{c}\text { decl. } \\
(\mathrm{J} 2000)\end{array}$ & $\begin{array}{c}\text { Exposure time } \\
(\# \times s)\end{array}$ & Instrument \\
\hline F1-E1-2008 & 2008 Oct 19 & $05: 35: 23.02$ & $-04: 46: 26.37$ & $3 \times 1200$ & Hectochelle \\
\hline F1-E2-2008 & 2008 Oct 21 & $05: 35: 23.02$ & $-04: 46: 26.37$ & $3 \times 1200$ & Hectochelle \\
\hline F2-E1-2008 & 2008 Oct 20 & $05: 35: 15.14$ & $-05: 15: 08.42$ & $3 \times 1200$ & Hectochelle \\
\hline F3-E1-2008 & 2008 Oct 19 & $05: 35: 13.17$ & $-05: 31: 44.51$ & $3 \times 1200$ & Hectochelle \\
\hline F3-E2-2008 & 2008 Oct 21 & $05: 35: 13.17$ & $-05: 31: 44.51$ & $3 \times 1200$ & Hectochelle \\
\hline F4-E1-2008 & 2008 Oct 18 & $05: 35: 07.48$ & $-05: 17: 32.75$ & $3 \times 1200$ & Hectochelle \\
\hline F4-E2-2008 & 2008 Oct 20 & $05: 35: 07.48$ & $-05: 17: 32.75$ & $3 \times 1200$ & Hectochelle \\
\hline F5-E1-2008 & 2008 Oct 18 & $05: 35: 22.22$ & $-06: 07: 13.73$ & $3 \times 1200$ & Hectochelle \\
\hline F5-E2-2008 & 2008 Oct 20 & $05: 35: 22.22$ & $-06: 07: 13.73$ & $3 \times 1200$ & Hectochelle \\
\hline F1-E1-2009 & 2009 Feb 14 & 05:35:09.15 & $-05: 20: 42.98$ & $3 \times 1200$ & Hectochelle \\
\hline F1-E2-2009 & 2009 Nov 03 & $05: 35: 06.94$ & $-05: 17: 36.21$ & $3 \times 1200$ & Hectochelle \\
\hline F1-E3-2009 & 2009 Dec 01 & 05:35:06.94 & $-05: 17: 36.21$ & $3 \times 1200$ & Hectochelle \\
\hline F1-E4-2009 & 2009 Dec 03 & $05: 35: 06.94$ & $-05: 17: 36.21$ & $3 \times 1200$ & Hectochelle \\
\hline F2-E1-2009 & 2009 Mar 14 & $05: 35: 14.69$ & $-05: 04: 58.26$ & $3 \times 1200$ & Hectochelle \\
\hline F3-E1-2009 & 2009 Dec 02 & $05: 34: 52.35$ & $-05: 54: 23.11$ & $3 \times 1200$ & Hectochelle \\
\hline F4-E1-2009 & 2009 Dec 02 & $05: 35: 26.82$ & $-06: 09: 44.54$ & $3 \times 1200$ & Hectochelle \\
\hline F5-E1-2009 & 2009 Dec 02 & 05:35:09.76 & $-05: 16: 54.04$ & $3 \times 1200$ & Hectochelle \\
\hline F6-E1-2009 & 2009 Dec 02 & $05: 35: 20.82$ & $-04: 49: 07.77$ & $3 \times 1200$ & Hectochelle \\
\hline $\mathrm{OA}$ & 2008 Nov 06 & 05:35:07.2 & $-05: 52: 14.2$ & $4 \times 1200$ & MIKE \\
\hline $\mathrm{OB}$ & 2008 Nov 07 & 05:35:00.0 & $-05: 25: 18.4$ & $4 \times 1200$ & MIKE \\
\hline $\mathrm{OC}$ & 2008 Nov 07 & $05: 35: 26.9$ & $-05: 13: 13.2$ & $4 \times 1200$ & MIKE \\
\hline OD & 2008 Nov 06 & $05: 35: 26.9$ & $-04: 47: 34.7$ & $5 \times 1200$ & MIKE \\
\hline OA1 & 2014 Feb 21 & $5: 35: 12.00$ & $-5: 30: 00.0$ & $6 \times 600$ & M2FS (Mg) \\
\hline OB1 & 2013 Dec 01 & $5: 35: 24.61$ & $-5: 11: 58.2$ & $4 \times 600$ & M2FS (Mg) \\
\hline OC1 & 2013 Nov 26 & $5: 35: 12.00$ & $-6: 00: 00.0$ & $4 \times 600$ & M2FS (Mg) \\
\hline OD1 & 2013 Nov 26 & $5: 35: 24.00$ & $-4: 45: 00.0$ & $5 \times 600$ & M2FS (Mg) \\
\hline NA & 2014 Feb 23 & $6: 40: 25.48$ & $+9: 48: 26.0$ & $5 \times 600$ & M2FS (Mg) \\
\hline NB & 2014 Feb 25 & $6: 41: 19.45$ & $+9: 30: 28.6$ & $5 \times 600$ & M2FS (Mg) \\
\hline LOA & 2014 Dec 18 & $5: 35: 12.00$ & $-5: 18: 04.0$ & $6 \times 600$ & M2FS (Li) \\
\hline LOB & 2014 Dec 21 & 5:35:09.00 & $-6: 02: 00.6$ & $3 \times 1200$ & M2FS (Li) \\
\hline LOC & 2014 Dec 24 & $5: 35: 22.90$ & $-4: 43: 27.8$ & $4 \times 1200$ & M2FS (Li) \\
\hline
\end{tabular}

Note.

${ }^{\text {a }}$ Data presented in T09 and T15 are not listed in this table.

cluster, it does suggest that sources containing $\mathrm{Li}$ I are at least casually related. No similar confirmation has been found for the sources in the ONC in T09.

In this paper, we revisit the published data, supplemented by more recent observations, to identify a more complete sample of the multiple stars in the ONC and NGC 2264, as well as to reexamine the kinematic structure of these regions. In Section 2, we present all of the additional data taken since the studies of T09 and T15. In Section 3, we discuss the construction of the final catalog and the identification of binary stars. Section 4 is focused on the specifics of the multiplicity in these regions, as well as the fitting of the orbits for select stars for which sufficient data were available. Meanwhile, Section 5 looks at the stellar velocity distribution. Finally, in Section 6, we summarize and discuss our findings.

\section{DATA}

We reanalyzed all of the spectra previously obtained by T09 for the ONC region and by T15 for the NGC 2264 region (including several stars observed but not included in their published catalog) using Hectochelle (Szentgyorgyi et al. 1998) and MIKE fibers (Bernstein et al. 2003; Walker et al. 2007). In addition to these data, we include new observations from these instruments and from the Michigan/Magellan Fiber System (M2FS; Mateo et al. 2012).

\section{1. $M 2 F S$}

M2FS is a multi-object spectrograph on the Magellan Clay Telescope that is capable of both low-resolution and highresolution echelle spectroscopy. Up to 256 targets can be observed over a $29^{\prime}$ field of view. The fibers observing these targets are split into two independent yet identical spectrographs. M2FS fibers need to be plugged in manually into predrilled plates. The minimum allowed separations between fibers is $12^{\prime \prime}$. A slitwidth of $180 \mu \mathrm{m}$ yields a typical resolution of $R \sim 20,000$.

We observed a total of four fields toward the ONC and two fields toward NGC 2264 in 2013 November and 2014 February (Table 1) using M2FS. We targeted a subset of stars from the T09 and T15 samples that was previously thought to be varying and/or had multiple reliable detections. The $\mathrm{Mg}$ I filter was used covering a wavelength range of $\sim 5100-5210 \AA$.

In addition, we also observed three fields toward the ONC in 2014 December with M2FS, but using the $\mathrm{H} \alpha$ and Li I filters covering range of $6525-6750 \AA$. Since two separate orders are observed simultaneously, only 128 targets can be observed in this configuration. We mainly obtained spectra for those sources that were originally identified by T09 as blueshifted relative to the gas in order to confirm their membership in the cluster via the presence of the $\mathrm{Li}$ I line, which can be used as an indicator of youth (Briceno et al. 1997). Additionally, given the 
available fibers, we observed objects that were previously monitored and had $V$ fluxes between 12 and 13.5 mag.

Data were reduced using a custom Python code written by $\mathrm{J}$. Bailey to merge the data and subtract the bias, and the IRAF pipeline HYDRA to trace the orders, extract the spectra, calculate and apply the wavelength solution using a set of ThAr exposures, and perform sky subtraction.

Spectra taken with the $\mathrm{Li}$ I and $\mathrm{H} \alpha$ filters were particularly affected by the strong nebular emission lines from $\mathrm{S}_{\text {II }}(6717$ and $6731 \AA$ ) and $\mathrm{N}_{\text {II }}(6549$ and $6583 \AA$ ), as well as H $\alpha$. These features are always narrow and appear in conjunction with each other in any given spectrum. They would be present as the only features even in the spectra of stars that were too faint to be detected. All of these features were masked out in the final data product if they were observed during the visual examination. However, because the $\mathrm{H} \alpha$ nebular line often interfered with the line that should have been observed due to stellar emission, often superimposed near the center of the line or barely offset from it, the masking process makes it impossible for us to reliably measure the equivalent widths of $\mathrm{H} \alpha$ for most spectra, and prevents us from detecting narrow stellar lines (both emission and absorption) in nearly all of the sources.

In addition to these narrow features, some spectra exhibited very broad and strong emission-like features at 6600 and $6725 \AA$ in $\mathrm{H} \alpha$ and Li I, respectively, spanning $\sim 20 \AA$ in width. These features appeared in approximately the same pixel range in both orders and are thought to be caused by Littrow ghosts from the optics. In the data taken with the Mg filter, a narrower and weaker feature appeared at $5181 \AA$ in the "blue" spectrograph, and at $5187 \AA$ in the "red" one. They are expected to have similar origins. In those cases where these features appeared to be significant, they were masked out.

\subsection{Hectochelle}

In addition to the T09 observations of the ONC made in 2007 with Hectochelle, data were also acquired in 2008 and 2009 (Table 1). The multi-fiber echelle spectrograph Hectochelle on the MMT has a $1^{\circ}$ field of view and can observe up to 240 targets simultaneously which can be positioned via robotic arms. The RV31 filter was used to cover the wavelength range $\sim 5150-5300 \AA$ with a typical resolution of $R \sim 35,000$. The data have been reduced using an IRAF pipeline developed by G. Fürész. A more detailed description of the Hectochelle data reduction can be found in Sicilia-Aguilar et al. (2006).

\section{3. $M I K E$}

The stars that were previously observed by T09 in ONC using MIKE fibers on the Magellan Clay telescope had an additional epoch observed in 2008 November (Table 1). MIKE consists of two independent spectrographs that can observe 128 fibers each. One of the spectrographs was used to cover the wavelength $\sim 5120-5190 \AA$, and the other one to cover $\sim 5140-5210 \AA$, at a resolution of $R \sim 18,000$. A description of the data reduction is available in T09.

\section{RV MEASUREMENTS}

All the data were processed through the IRAF package RVSAO (Kurtz \& Mink 1998) in order to extract the RVs from all of the targets by performing cross-correlation against the synthetic stellar spectroscopic templates of Munari et al. (2005). As in T09, all of the templates had surface gravity $\log (g)=3.5$, effective temperatures $\left(T_{\text {eff }}\right)$ between 3500 and $7000 \mathrm{~K}$ in steps of $250 \mathrm{~K}$, and solar metallicity. The previously reported spectra by $\mathrm{T} 09$ and $\mathrm{T} 15$ have also been re-correlated to achieve a homogeneous sample.

Default filtering parameters (low_bin $=5$, top_low $=20$, top_nrun $=125$, nrun $=255$ ) were used during cross correlation to filter noise and large-scale structure in the spectra. However, rapidly rotating stars have broad and occasionally overlapping lines which would not be effectively processed with these parameters. For such objects, different filtering parameters (low_bin $=3$, top_low $=10$ ) were used if the uncertainty from the revised correlation was no greater than $0.05 \mathrm{~km} \mathrm{~s}^{-1}$ and the resulting measure of the signal-to-noise $R$ value was greater:

$$
R=2^{-\frac{1}{2}} h \sigma_{a}^{-1}
$$

where $h$ is the height of the peak of the correlation function, and $\sigma_{a}$ is the error estimated from the rms of the asymmetric component of the correlation (Tonry \& Davis 1979).

In data taken in 2014 December, the $\mathrm{Li}$ I and $\mathrm{H} \alpha$ orders have been cross-correlated separately. The $\mathrm{H} \alpha$ line is the strongest feature in its order and the shape of the peak of the cross correlation is largely driven by the shape of this line. The $\mathrm{H} \alpha$ line is usually much wider than rotational velocity broadening and, because it is not photospheric, it can be affected by chromospheric motions. Additionally, because the center of the line was typically masked to remove scattered light, the velocities obtained from this order are inherintly more uncertain than those obtained from the $\mathrm{Li}$ I order. If the velocities obtained from both orders differed by less than the uncertainties added in quadrature, then the average velocity and uncertainy ( $v_{\text {ave }}$ and $\sigma_{\text {ave }}$ ) were calculated via the varianceweighted mean, and the $R$ value was added in quadrature. This could only be done for $43 \%$ of the sources. If no reasonable cross-correlation could be achieved from the $\mathrm{Li}$ I order, then $\mathrm{H} \alpha$ velocities were used ( $9 \%$ of the sources).

Typical uncertainties for the individual RV measurements are $0.8 \mathrm{~km} \mathrm{~s}^{-1}$ in NGC 2264 and $1.2 \mathrm{~km} \mathrm{~s}^{-1}$ in the ONC. Weighted average uncertainties for individual stars are $0.4 \mathrm{~km} \mathrm{~s}^{-1}$ in NGC 2264 and $0.7 \mathrm{~km} \mathrm{~s}^{-1}$ in the ONC. It is possible that the larger uncertainties in the measurements toward the ONC are due to higher extinction.

When constructing the table of all of the available measurements for all of the sources, we retained only those measurements that had $R>3$ and $-100<\mathrm{RV}<100 \mathrm{~km} \mathrm{~s}^{-1}$. After that, the time series of the measured velocities for each object was visually examined for inconsistent data. Common issues that were noted were as follows.

1. Since the MIKE and M2FS fiber plates had to be drawn and plugged manually, a wrong star would occasionally be observed; this is found when the matched template for one or more is wildly different and would also exhibit velocity unlike the remaining observations of the same target.

2. Measurements with $3<R<6$ could be inconsistent with the remaining data for the target; this is likely a result of poor signal to noise that was not caught through automatic filtering.

3. Many of the measurements taken on 2009 February 12 with Hectochelle appear to be contaminated by moonlight; despite having a high $R$ value, these would typically 
Table 2

Sources Showing No Variation in Radial Velocity Between Multiple Observations ${ }^{\mathrm{a}}$

\begin{tabular}{|c|c|c|c|c|c|c|c|c|c|}
\hline $\begin{array}{l}\mathrm{RV} \\
\#\end{array}$ & $\begin{array}{c}\text { R.A. } \\
\text { (J2000) }\end{array}$ & $\begin{array}{l}\text { Decl. } \\
(\mathrm{J} 2000)\end{array}$ & $\begin{array}{l}\text { Date } \\
\text { (JD) }\end{array}$ & $\begin{array}{c}v \\
\left(\mathrm{~km} \mathrm{~s}^{-1}\right)\end{array}$ & $\begin{array}{c}\sigma \\
\left(\mathrm{km} \mathrm{s}^{-1}\right)\end{array}$ & $R$ & $\begin{array}{l}\text { Temp } \\
(\mathrm{K})\end{array}$ & $\overline{R R} ?^{\mathrm{b}}$ & $\overline{\text { Instrument }}$ \\
\hline$\overline{1}$ & 05:33:17.95 & $-05: 21: 38.6$ & $\begin{array}{l}2454401.0 \\
2454401.8 \\
2454757.9 \\
2454760.0 \\
2454876.6\end{array}$ & $\begin{array}{l}29.17 \\
30.38 \\
23.64 \\
25.86 \\
24.78\end{array}$ & $\begin{array}{l}4.51 \\
4.55 \\
3.14 \\
3.88 \\
2.58\end{array}$ & $\begin{array}{l}3.26 \\
3.07 \\
6.05 \\
5.63 \\
7.42\end{array}$ & $\begin{array}{l}3500 \\
3750 \\
3750 \\
3750 \\
3750\end{array}$ & $\begin{array}{l}\ldots \\
\cdots \\
\cdots \\
\cdots \\
\cdots\end{array}$ & $\begin{array}{l}\text { Hectochelle } \\
\text { Hectochelle } \\
\text { Hectochelle } \\
\text { Hectochelle } \\
\text { Hectochelle }\end{array}$ \\
\hline 2 & 05:33:20.44 & $-05: 11: 24.0$ & $\begin{array}{l}2454757.9 \\
2454760.0 \\
2454904.6 \\
2455167.9\end{array}$ & $\begin{array}{l}25.83 \\
26.79 \\
24.57 \\
31.86\end{array}$ & $\begin{array}{l}1.07 \\
1.18 \\
1.12 \\
1.43\end{array}$ & $\begin{array}{r}12.19 \\
13.83 \\
14.51 \\
8.73\end{array}$ & $\begin{array}{l}4000 \\
4000 \\
4000 \\
4000\end{array}$ & $\begin{array}{l}\ldots \\
\mathrm{y} \\
\mathrm{y} \\
\mathrm{y}\end{array}$ & $\begin{array}{l}\text { Hectochelle } \\
\text { Hectochelle } \\
\text { Hectochelle } \\
\text { Hectochelle }\end{array}$ \\
\hline
\end{tabular}

Notes.

${ }^{a}$ Includes sources with insufficient number of detections to determine variability.

b Measurement was processed with low_bin $=3$, top_low $=10$.

${ }^{\mathrm{c}} \mathrm{RV}$ 1-1154 belongs to the ONC, RV 1155-2057 to NGC 2264.

(This table is available in its entirety in machine-readable form.)

Table 3

Sources with Variable Radial Velocity

\begin{tabular}{|c|c|c|c|c|c|c|c|c|c|c|c|}
\hline $\begin{array}{l}\mathrm{RV} \\
\#\end{array}$ & $\begin{array}{c}\text { R.A. } \\
(\mathrm{J} 2000)\end{array}$ & $\begin{array}{c}\text { Decl. } \\
(\mathrm{J} 2000)\end{array}$ & $\begin{array}{l}\text { Date } \\
\text { (JD) }\end{array}$ & $\begin{array}{c}v_{1} \\
\left(\mathrm{~km} \mathrm{~s}^{-1}\right)\end{array}$ & $\begin{array}{c}\sigma_{1} \\
\left(\mathrm{~km} \mathrm{~s}^{-1}\right)\end{array}$ & $\begin{array}{c}v_{2}^{\mathrm{a}} \\
\left(\mathrm{km} \mathrm{s}^{-1}\right)\end{array}$ & $\begin{array}{c}\sigma_{2} \\
\left(\mathrm{~km} \mathrm{~s}^{-1}\right)\end{array}$ & $R$ & $\begin{array}{c}\text { Temp } \\
(\mathrm{K})\end{array}$ & $\mathrm{RR} ?^{\mathrm{b}}$ & Instrument \\
\hline \multirow[t]{6}{*}{$10^{\mathrm{c}}$} & $05: 33: 29.38$ & $-05: 07: 49.1$ & 2454401.0 & 31.39 & 1.95 & $\ldots$ & $\ldots$ & 8.24 & 5000 & $\ldots$ & Hectochelle \\
\hline & & & 2454401.8 & 29.55 & 1.66 & $\ldots$ & $\ldots$ & 10.62 & 4750 & $\ldots$ & Hectochelle \\
\hline & & & 2454757.9 & 30.34 & 1.44 & $\ldots$ & $\ldots$ & 11.24 & 5000 & $\ldots$ & Hectochelle \\
\hline & & & 2454760.0 & 29.20 & 1.31 & $\ldots$ & $\ldots$ & 12.47 & 4250 & $\ldots$ & Hectochelle \\
\hline & & & 2455138.8 & 19.19 & 1.01 & $\ldots$ & $\ldots$ & 13.12 & 5000 & $\ldots$ & Hectochelle \\
\hline & & & 2455168.9 & 32.64 & 1.46 & $\ldots$ & $\ldots$ & 10.28 & 4250 & $\ldots$ & Hectochelle \\
\hline \multirow[t]{3}{*}{26} & $05: 33: 36.37$ & $-05: 01: 40.5$ & 2454759.9 & 99.50 & 2.40 & $\ldots$ & $\ldots$ & 12.37 & 5750 & $\ldots$ & Hectochelle \\
\hline & & & 2455138.8 & 20.43 & 1.32 & $\ldots$ & $\ldots$ & 10.17 & 5000 & $\ldots$ & Hectochelle \\
\hline & & & 2455166.9 & 72.24 & 6.11 & $\ldots$ & $\ldots$ & 4.43 & 5500 & $\ldots$ & Hectochelle \\
\hline
\end{tabular}

Notes.

${ }^{\text {a }}$ Velocity obtained from the second peak of the cross-correlation for double-lined binaries.

b Measurement was processed with low_bin $=3$, top_low $=10$.

${ }^{c}$ Have at least $4 R>6$ measurements separated by more than 3 days with only a single variable velocity.

(This table is available in its entirety in machine-readable form.)

have an RV uncorrected for a barycentric motion of $\sim 0 \mathrm{~km} \mathrm{~s}^{-1}$.

Contamination from these measurements is one of the main reasons for the discrepancy between the results presented in this paper and those in T09. These measurements have been removed from the final table and are not considered in any of the calculations. Any velocity measurement with $3<R<6$ that remained in the table was excluded from the following calculations described in this section as well, but they have been used only in visual examination of the data to confirm the presence of the variability and in fitting the orbits of the identified binaries (Section 4). Beyond removing contaminating data, no velocity zero-point offset was applied to the data taken on different days, as there appeares to be almost no systematic variability between the different epochs. The median offset of individual measurements relative to the $v_{\text {ave }}$ of a given star within each epoch is typically within $0.3 \mathrm{~km} \mathrm{~s}^{-1}$ and less than $1 \mathrm{~km} \mathrm{~s}^{-1}$, which is consistent with the measured uncertainties.

We use the reduced $\chi^{2}$ as a measure of the consistency of the velocity in the time series. We identify systems as RV variable if they have reduced $\chi^{2}>16(\sim 4 \sigma)$. In total, there are 2057 sources with at least a single velocity measurement with $R>6$, of which 1154 are found toward ONC and 903 toward NGC 2264 (Table 2). A total of 130 sources have been identified as $\mathrm{RV}$ variable, with 79 toward the ONC region and 51 toward NGC 2264 (Table 3). Individual measurements of all of the non-variable sources are reported in Table 2. All of the sources identified as RV variable are listed in the Table 3.

\section{MULTIPLICITY}

\subsection{Measured Multiplicity Fraction}

Out of the 137 sources originally identified by T09 as RV variables in the ONC region, we can confirm only 15 as such in our final catalog. The remaining sources exhibited either little to no change in velocity or had variable velocity measurements that were of low significance. Some of those sources could still be multiple systems, but we do not consider them further here due to our stricter limits for significance to avoid false positives. Upon closer examination, those sources that were previously identified as double-lined binaries by the presence of the second 

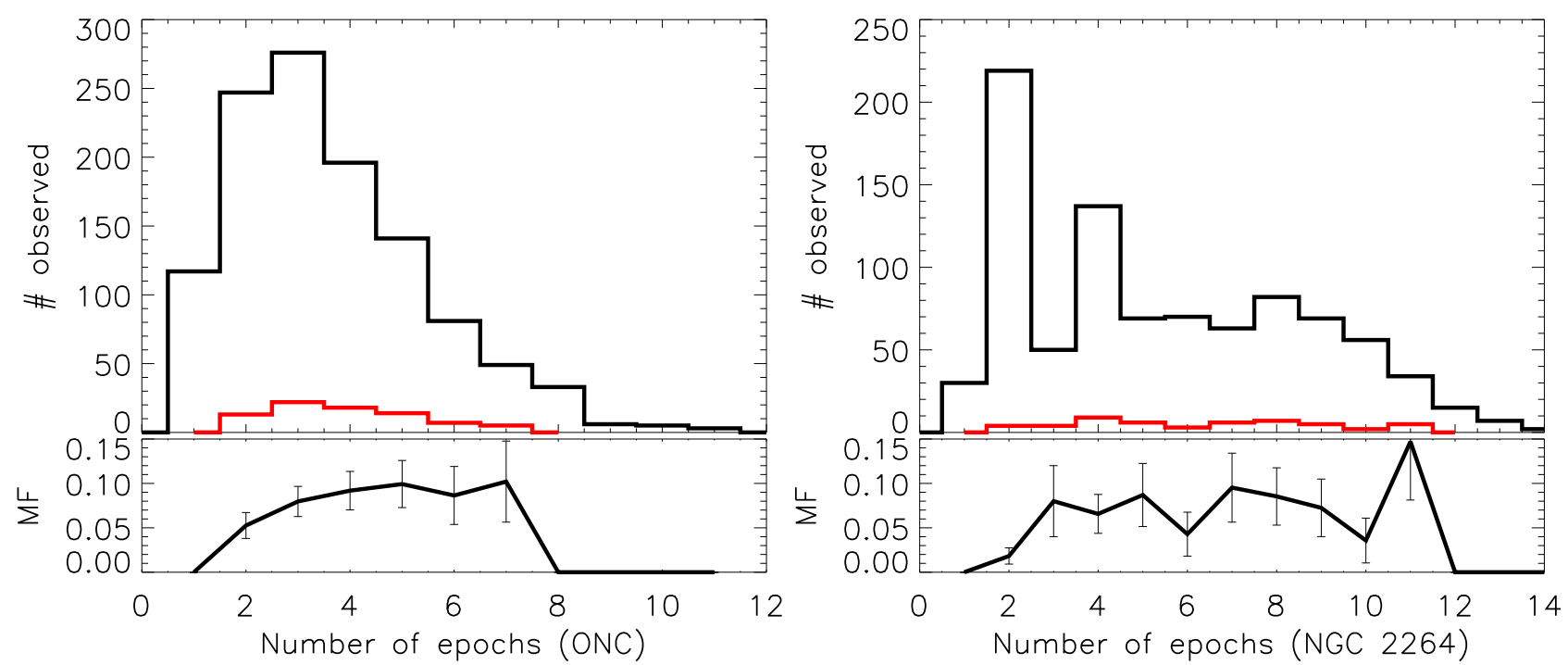

Figure 1. Number of observations made for all of the objects in the sample is shown in black. Red shows only those sources with $\chi^{2}>16$. The bottom section shows the ratio of the number of sources with $\chi^{2}>16$ per number of all sources with a given number of epochs observed. Left panel: ONC. Right panel: NGC 2264 .

peak in the correlation either could not be confirmed as such or were flagged as variable by our method. For this reason, we focus only on the 130 sources that we can identify as only RV variables with either new or reanalyzed spectra.

We consider two possible causes of the RV variability. First, it could be due to the orbital motion of the muliple systems. Second, the changing RV could be a result of RV jitter due to spots on the surface of magnetically active stars. The typical effect of jitter in main-sequence stars is on the order of a few $\mathrm{m} \mathrm{s}^{-1}$ (Hillenbrand et al. 2015), but it could be on the order of $\sim 1 \mathrm{~km} \mathrm{~s}^{-1}$ in pre-main-sequence stars (Donati et al. 2013, 2014, 2015).

Given that RV variable sources typically had significant fluctuation from $v_{\text {ave }}, \mathrm{RV}$ jitter can only account for a handful of sources. We identify sources as multiple if they have at least one measurement $\left|v_{\text {ave }}-v\right|>4 \mathrm{~km} \mathrm{~s}^{-1}$. A total of 113 sources satisfy this requirment. The remaining 17 sources fall below this threshold; while they do exhibit RV variability, we cannot confirm that it is due to orbital motion within a multiple system. Because of this, we exlcude them from any calculations involving the multiplicity fraction.

The velocity curves for most of our sources are very undersampled, and so we could fail to detect real RV variables. We consider the minimum number of epochs required for a guaranteed detection of an RV varying system to be three. With only 2 measurements, it is possible to miss a binary system due to coincidental timing between the observations. However, with three or more epochs of data, the fraction of the number of systems identified as multiples to the total number of stars remains relatively unchanged (Figure 1).

We attempted to identify false positive sources that were flagged as multiples if they had only a single discrepent measurement from the mean. To do that, we calculated the reduced $\chi^{2}$ while thowing out the single most variable RV measure from $v_{\text {ave }}$ and identified sources where this revised reduced $\chi^{2}<16$. We required that (a) all of the sources flagged by this method have at least four measurements with $R>6$, since otherwise the lack of detected variability could be due to poor sampling; (b) at least four epochs during which the measurements were taked are separated in time by more than

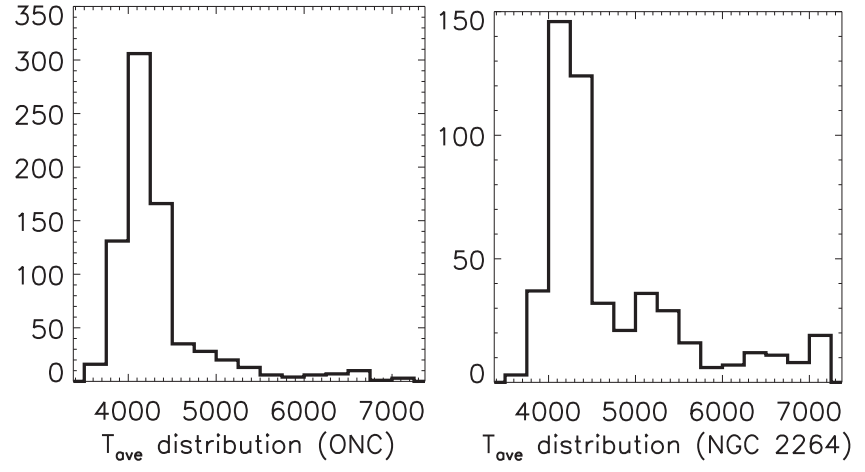

Figure 2. Average effective temperature disribution observed toward stars in ONC and NGC 2264.

two days and, as such, short separations in time cannot detect variability due to orbital motion with orbits longer than a few days; and (c) only a single measurement has a discrepent RV, including $R<6$ measurements if they have comparable variability from $v_{\text {ave }}$. This identified six sources: RV 10, 138, $929,1372,1496$, and 1660. In the cases of RV 1372, 1496, and 1660, we provide detailed orbital fits (Section 4.3). These fits allow little doubt as to the nature of these sources as muliple systems; however, without a single strongly deviating RV they would not have been identified as such based on the data in this paper. For this reason, while the remaining three sources (RV 10, 138, and 929) are flagged in Table 3 as possible false positives, we include them in the flollowing calculations.

The observed multiplicity fraction (defined as the overall number of the multiple systems, hereafter MF) within the RV data set is $8.0 \pm 1.2 \%$ toward the $\mathrm{ONC}$ and $6.7 \pm 1.1$ toward NGC 2264 if we include all of the sources in the Tables 2 and 3 . However, if we require $v_{\text {ave }}$, converted from the heliocentric to local standard of rest (lsr) reference frame (Kerr \& LyndenBell 1986), to range from -5 to $20 \mathrm{~km} \mathrm{~s}^{-1}$ for both single stars and binaries to limit the contamination from the sources that are not the members of these clusters (T15, also more in Section 5), then MF becomes $5.8 \pm 1.1 \%$ for ONC (30 multiples out of 518 stars observed) and $5.3 \pm 1.2 \%$ for NGC 2264 (21 out of 397). Uncertainties were obtained as $N_{\text {multiple }}^{\frac{1}{2}} N_{\text {total }}^{-1}$. 

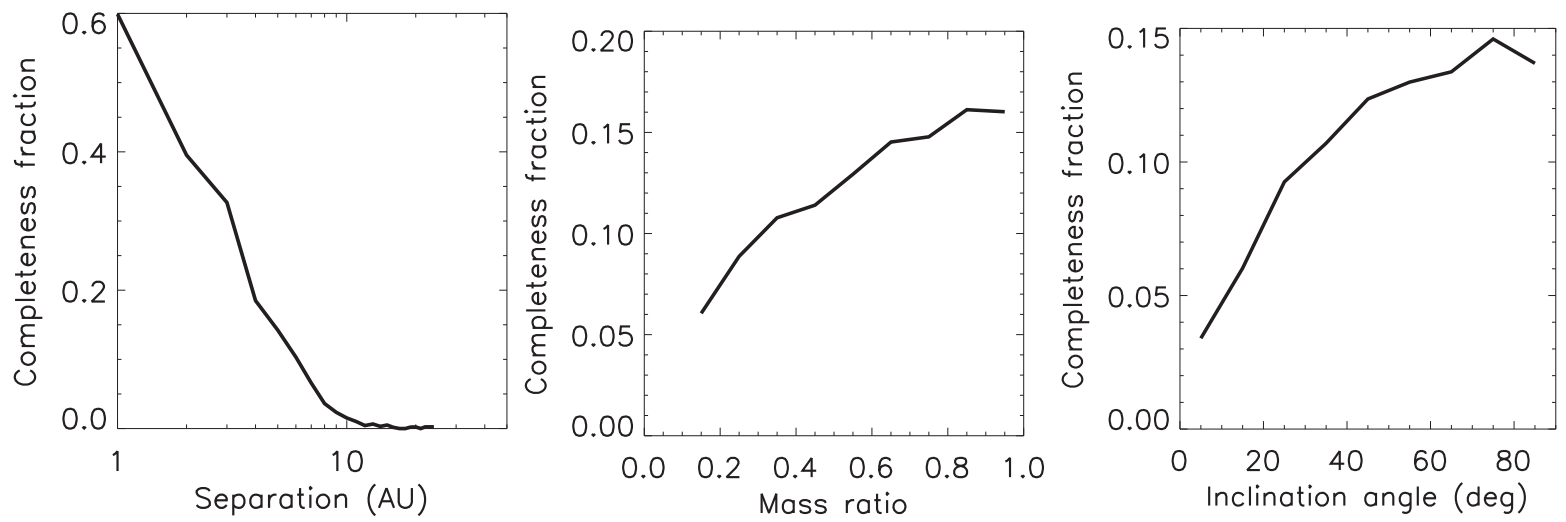

Figure 3. Completeness limits of the detection of the multiple systems depending on their orbital parameters.

\subsection{Comparison to the Field}

To compare the MF that we observe toward ONC and NGC 2264 to that observed in the field, we ran a Monte-Carlo simulation producing a synthetic field population that would be consistent with the distribution of binary properties in the nearby $\mathrm{G}$ dwarfs (Raghavan et al. 2010). For each of the stars in our sample that had $-5<v_{\mathrm{lsr}}<20 \mathrm{~km} \mathrm{~s}^{-1}$ and $>3$ detections (i.e., those from which the MF was measured), we generated randomly configured systems (including both single and multiple) that had the same dates of observation and the same uncertainties as the data. We then ran the same detection test that would identify binary systems from the generated population, producing an MF for a single test case. This process was repeated 1000 times. The average MF from all of the test cases, both in the ONC and NGC 2264 regions, was determined separately. Uncertainty was determined from the $1 \sigma$ dispersion in the generated MF between the 1000 test cases.

For the ONC, there are 518 systems that make up the population from which the $\mathrm{MF}$ was estimated. Of these systems, $\sim 120$ stars have uncertainties too large to be detected as variable in any of the runs. The average MF of the synthetic population is $4.8 \pm 0.9 \%$. NGC 2264 had a total of 397 systems, of which $\sim 70$ cannot be identified as multiples in any run, and an average MF of $6.1 \pm 1.2 \%$.

The estimate of the expected MF makes an implicit assumption concerning the mass of the primary stars. To simulate the population of the field stars to be consistent with what was measured by Raghavan et al. (2010), $1 M_{\odot}$ primaries were chosen. However, the masses of the stars monitored in these clusters are expected to be significantly lower. The typical effective temperature template matched toward these stars is $4000 \mathrm{~K}$ (Figure 2). From this, we can estimate them to be $\mathrm{K}$ stars with typical masses on the order of $0.7 M_{\odot}$ (Baraffe et al. 2015). Stellar multiplicity varies strongly with stellar mass, and lower-mass stars have been found to have lower MF than higher-mass stars (Fischer \& Marcy 1992; Duchêne \& Kraus 2013). G stars have typically been used for comparison because surveys of their orbital parameters have been by far the most comprehensive.

In addition, because the mass of the primary is lowered, the distribution of the masses of the secondary is also cut off at smaller values. Because of this, the difference in the peaks of the RV fluctuations due to the presence of a companion would become smaller as well; therefore, fewer systems would be detected. By generating a population with identical orbital parameters around $0.7 M_{\odot}$, we instead infer MFs of $4.5 \pm 0.9 \%$ for ONC and $5.8 \pm 1.2 \%$ for NGC 2264 .

The dispersion in the MF generated with identical orbital parameters between separate runs (all consisting of 1000 test cases) observed with the cadence and uncertainties set by the same population is typically $<0.1 \%$. To estimate the systematic effects due to a potentially different distribution of periods and overall number of binary systems in these clusters compared to what was previously found in the field, we varied one parameter at a time and looked for a difference in MF. Varying the underlying binarity fraction by $2 \%$ ( $1 \sigma$ values quoted by Raghavan et al. 2010) typically changes the extracted MF by $0.2 \%$. No uncertainties on the orbital parameters were made apparent, although varying the peak of the period distribution by $0.1 \log P$ (where $P$ is measured in days) produces an $\mathrm{MF}$ that is different by $0.5 \%$ when the average period is decreased from 293 to 233 years, and by $0.3 \%$ when the period is increased to 369 years. Varying the standard deviation of the period distribution by $0.1 \sigma_{\log P}$ changed the MF by $0.4 \%$. Changing the mass ratio and eccentricity from uniformly distributed to those that are described by Duquennoy \& Mayor (1991) decreases the MF by $0.5 \%$ and $0.4 \%$, respectively.

To determine the completeness limits that we probe with these observations, we recorded orbital parameters from all of the generated binary systems and determined a fraction that would be detectable relative to all of the binaries that satisfy the specific orbital parameters (Figure 3). Unsurprisingly, the most easily detectable systems have short orbital periods, comparable masses between the primary and the secondary, and an edge-on orientation. It is possible to detect only $60 \%$ of all of the systems with separations $<1$ au because either the other orbital parameters make a detection difficult or the uncertainty in the measurements that were applied to the generated velocity curves were too large for a reliable detection. Beyond the separations of $15 \mathrm{au}$, there are almost no systems that could be detected as binaries based on their RV variability. Combining all of the possible separations and inclination angles, only $15 \%$ of multiple systems can be detected for stars with a mass ratio on the order of unity (in this case both stars have mass of 1 $\left.M_{\odot}\right)$; however, this rapidly decreases to $\sim 5 \%$ for those companions with only $0.2 M_{\odot}$.

\subsection{Orbital Parameters}

Individual cross-correlations of all of the systems identified as binaries were visually examined to determine whether or not it is possible to see a second peak due to the presence of the 
second star. We required that any star flagged as a double-lined binary must exhibit multiple peaks or a skewed correlation function in at least two epochs to minimize spurious detections. There were a total of 15 of such systems, of which 10 were found toward NGC 2264 and 5 toward ONC. In epochs where it was possible, a Gaussian was fitted to both peaks to find the velocities of both components. We assigned uncertainties to these measurements of $2 \mathrm{~km} \mathrm{~s}^{-1}$, limited by the resolution of the extracted cross-correlations function. All of these measurements are included in Table 3.

Out of 130 sources flagged as binaries in this paper, 6 singlelined binaries have $\geqslant 10 \mathrm{RV}$ measurements and 2 double-lined binaries have $\geqslant 9$ measurements. We attempted to fit the velocity curves of these sources as they contained a sufficient number of measurements and redundancy on the measurements to obtain a unique solution. Because sources in NGC 2264 have been monitored more frequently than those in ONC (Figure 1), all 8 of these sources are found toward NGC 2264.

To fit the orbits, the IDL package RVFIT (Iglesias-Marzoa et al. 2015) was used, which fits the following quantities: $P$ (the orbital period), $T_{P}$ (the time of periastron passage), $e$ (the eccentricity), $\omega$ (the argument of the periastron), $\gamma$ (the systemic velocity of the system), and $K_{1}$ (the amplitude of the RV fluctuation) and $K_{2}$ (the amplitude of the RV fluctuation for the second star if the system in question is a double-lined spectroscopic binary.) The derived quantities are the semimajor axis $a_{1} \sin i$ and the binary mass function $f . M \sin i$ contributions of the individual stars can be distinguished for double-lined systems.

RV 1768 showed the second peak in the correlation in 7 out of 11 epochs, and both peaks exhibited a similar strength. The remaining 4 epochs had measurements very close to the mean of the remaining measurements. Because we are unable to distinguish between the contributions of individual components, there could be a larger spread in the velocity due to line blending; to those measurements we assign an uncertainty of $5 \mathrm{~km} \mathrm{~s}^{-1}$.

As RV 1768 appeared to have almost equal mass in both components, there was some difficulty in distinguishing which of the two lines in a given epoch belonged to which star in cases of resolved measurements, and the velocity of which star was the most dominant in cases of unresolved measurements. An attemp was made for all of the perturbations of the line combinations to be fitted and examined by eye-the combination which provided the best fit is listed in Table 3 and the resulting fit is shown in Figure 4 and Table 5. The masses of the individual components $\left(\mathrm{M} \sin ^{3} i\right)$ are $\sim 0.25 M_{\odot}$. Given that the spectra for this system are best fit by a $\sim 4000 \mathrm{~K}$ template, this is a reasonable fit requiring only a modest inclination angle.

RV 1659 had a double-peaked correlation function in 8 out of 9 epochs, with one component clearly dominating over the other. It was best fit by a circular orbit, a period of 15.3 days, and $\mathrm{M} \sin ^{3} i$ of 0.73 and $0.58 M_{\odot}$ (Figure 5, Table 5). For orbits with no eccentricity, the argument of periastron carries no meaningful information.

Fitting information on the six single-lined systems is presented in Table 6 (Figures 6-11). Based on the characteristic heliocentric velocity of RV 1372 of $-32 \mathrm{~km} \mathrm{~s}^{-1}\left(\mathrm{v}_{\mathrm{lsr}} \sim\right.$ $-47 \mathrm{~km} \mathrm{~s}^{-1}$ ), it is unclear whether or not it is a member of NGC 2264. However, this system is typically best fit by a $4250 \mathrm{~K}$ template and has near-infrared fluxes of $J=12.270$,
$H=11.718$, and $K=11.464$ (Cutri et al. 2003). While there is some contamination in the fluxes from the companion, they are in a good agreement with those of other binary systems which follow the same templates (e.g., RV 1550, a system for which the orbital fit is also available and which has a systematic velocity that is consistent with the cluster mean, has $J=$ 12.262, $H=11.566, K=11.423$ ), making it likely that $\mathrm{RV}$ 1372 is not a foreground or background system, but rather that it was ejected from the cluster.

RV 1753 was previously monitored for change in RV by Karnath et al. (2013) over a period of 20 years with 35 epochs. All of the fitted orbital parameters from that study are in excellent agreement with the fits presented in this paper.

\section{VELOCITY STRUCTURE}

\section{1. $O N C$}

Some of the analysis of the ONC region performed in T09 was affected by contamination from moonlight, and the lowest signal-to-noise data also added scatter. While the overall conclusions of T09 remain unchanged, the sample presented in this paper has higher quality velocity measurements. For this reason, once again, we look at the relation between stellar RVs and ${ }^{13} \mathrm{CO}$ gas (Figures 12 and 13). All of the velocities in the plots are in the kinematic LSR reference frame to match that of the gas (Bally et al. 1987). To confirm that no binary stars contaminate the sample, we required that there be no variability in velocities with at least 3 epochs of observations. Unlike in T09, the peak of the RV distribution for stars follows the gas with no offset and a comparable velocity dispersion of $\sim 2.5 \mathrm{~km} \mathrm{~s}^{-1}$. The only exception to this is the $-5^{\circ} .5<\delta<-5^{\circ} .0$ range, which coincides with the location of inner ONC regions such as Trapezium and OMC 2/3. A recent paper by Da Rio et al. (2015) measuring the RV from the infrared spectra in Orion A also found a lack of a blueshifted tail extending beyond $v_{\mathrm{lsr}}<0 \mathrm{~km} \mathrm{~s}^{-1}$.

However, while not quite as pronounced as reported by $\mathrm{T} 09$, we do observe some blueshifted tail component in the stellar RVs relative to the ${ }^{13} \mathrm{CO}$ motions. To determine whether or not the sources that populate that tail are members of the cluster or unrelated foreground or background stars, we searched for Li I detection toward some of them (Table 4, Figure 12) as a signature of their youth to establish whether or not these sources could be causally related to the ONC. Overall, many of the sources that occupy the same velocity space as the gas and blueshifted tail were indeed found to contain Li I. While there is some contamination from sources that appear to be somewhat more evolved, their low numbers alone cannot account for the entirety of the blueshifted tail. On the other hand, nearly all of the sources that occupy the velocity space outside what is presented in Figure 12 lack in Li I.

There are several possible explanations for this tail. There could be a separate foreground population of young stars that is not an immediate part of the ONC. Alves \& Bouy (2012) and Bouy et al. (2014) argue that NGC 1980 is an example of such a foreground cluster. Unfortunately, it is located at $83^{\circ} .7<\alpha<83^{\circ} .9$ and $-6^{\circ} .1<\delta<-5^{\circ} .8$, and the presence of the blueshifted population in that region is minimal and not spatially coherent. This is consistent with what has been found by Da Rio et al. (2015). However, south of it, the ONC is starting to turn into the L1641 cloud, and thus it is possible that the tail in the southernmost regions can be attributed to this. 
The Astrophysical Journal, 821:8 (12pp), 2016 April 10

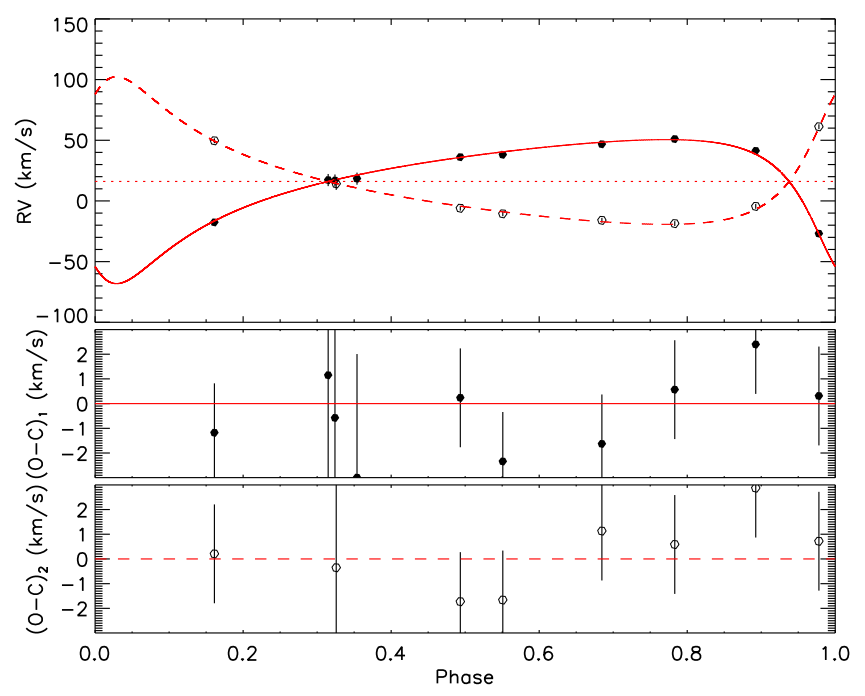

Figure 4. Orbital fit for RV 1768.

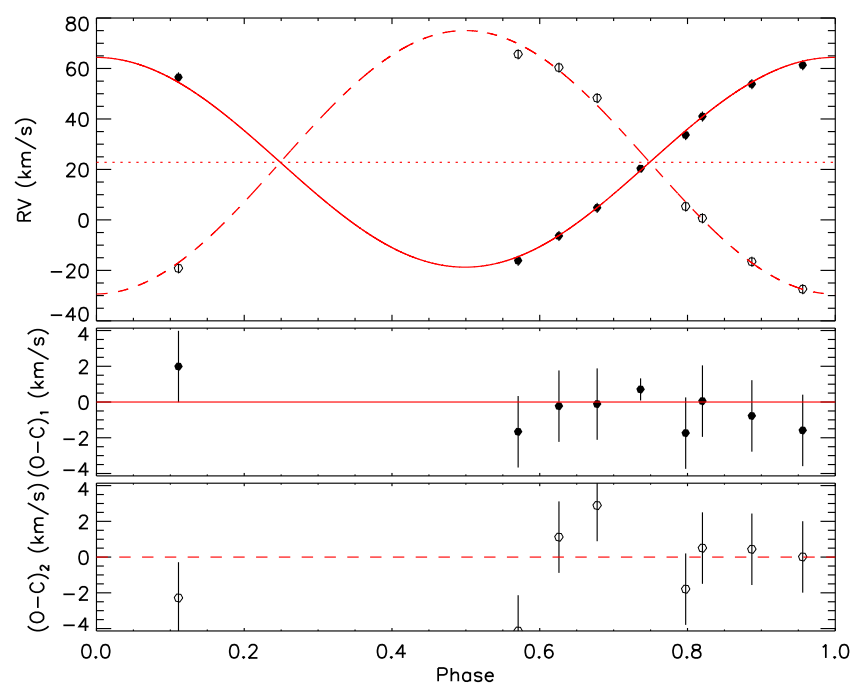

Figure 5. Orbital fit for RV 1659.

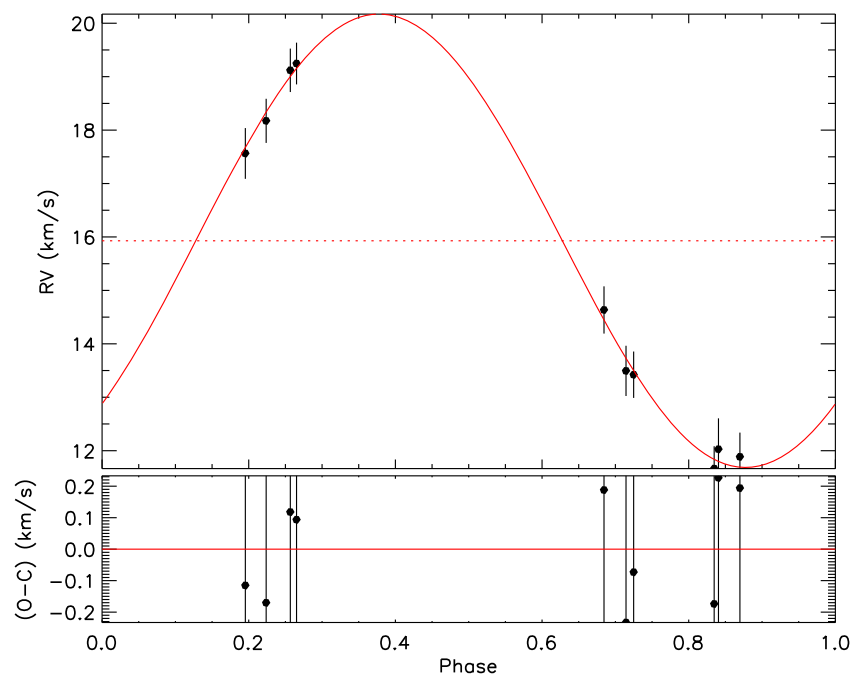

Figure 6. Orbital fit for RV 1166.

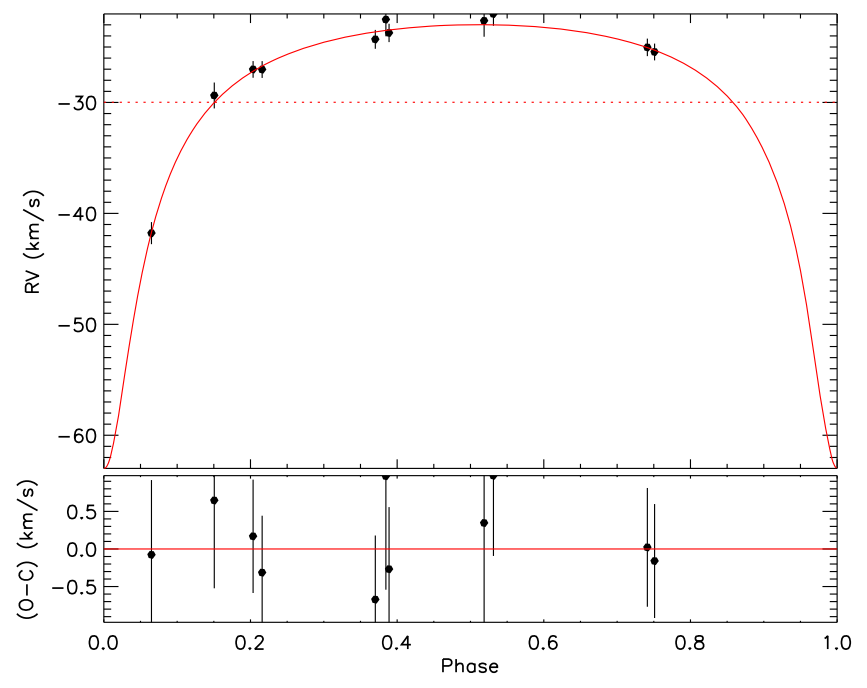

Figure 7. Orbital fit for RV 1372.

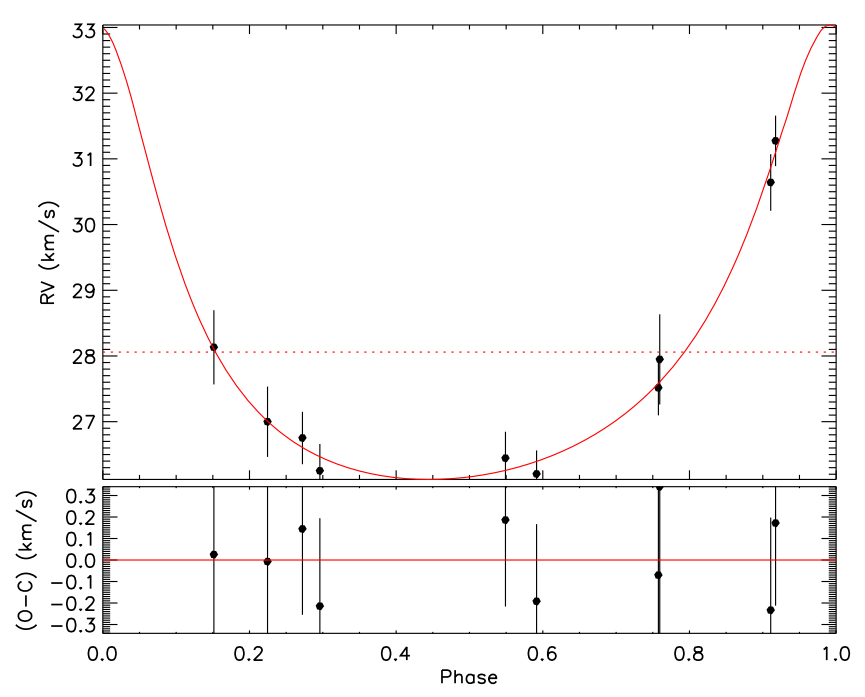

Figure 8. Orbital fit for RV 1496.

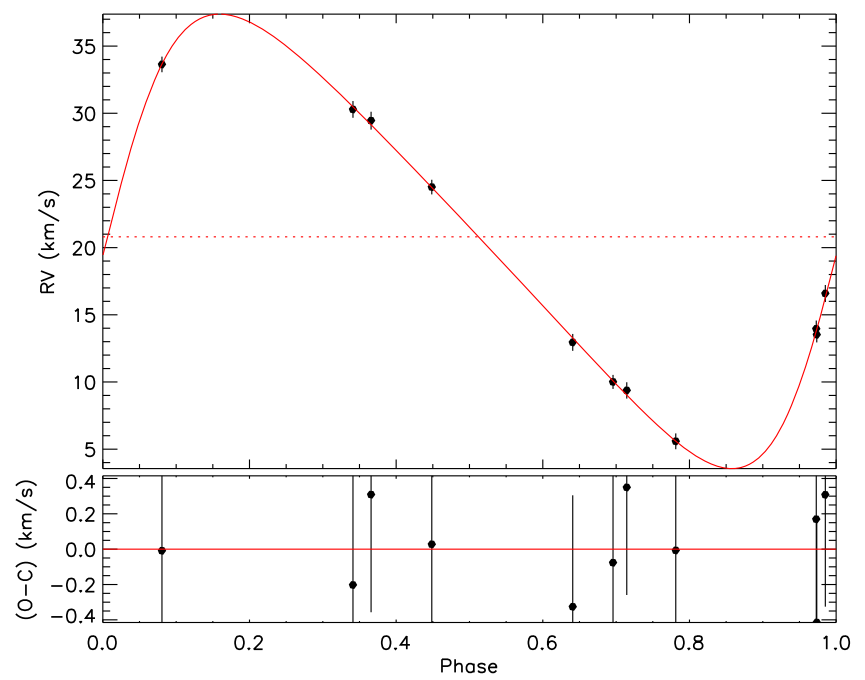

Figure 9. Orbital fit for RV 1550. 


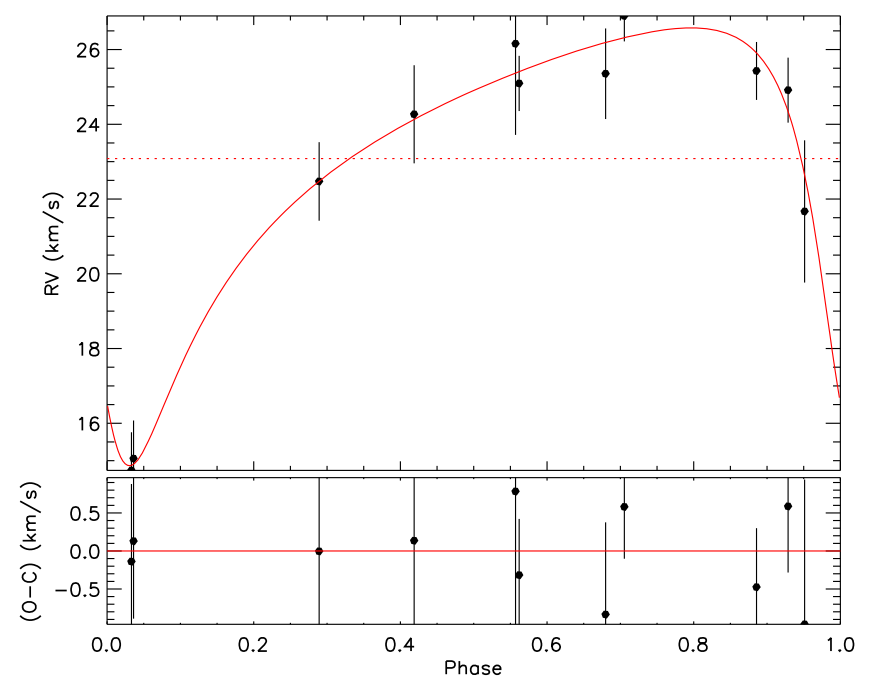

Figure 10. Orbital fit for RV 1660.

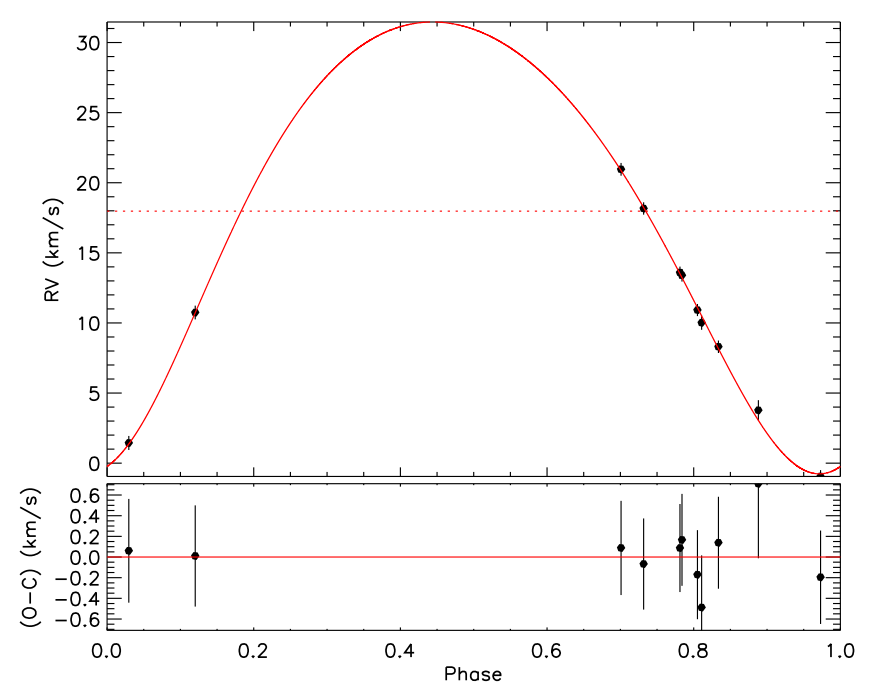

Figure 11. Orbital fit for RV 1753.

Some of these stars could have been dynamically scattered to achieve these velocities. While it is difficult to explain why there is no redshifted population to make the velocity distribution symmetric, it is possible that high extinction prevents us from observing it. The inner ONC is where this effect would be the most pronounced. Not only does this region have significantly higher stellar density than the rest of the cluster, allowing for more significant dynamical interactions between stars, but it is also more affected by extinction due to the high density of gas. It is possible that this can account for some of the observed blueshifted sources.

Alternatively, it is possible that the gas was being blown away by stellar feedback, leaving a somewhat older population of stars behind while newer stars formed. As suggested by T09, it is likely that in the northmost region, in the vicinity of NGC 1977, gas has been pushed back by irradiation from HD 37018 , HD 37077, and HD 36958, which are B1V, B3V, and B3V stars, respectively, leaving behind a mini-cluster.

Proszkow et al. (2009) and T09 instead postulate that the redshift in the gas in Trapezium and OMC $2 / 3$ could be due to the gravitational infall of the OMC $2 / 3$ filament toward the Trapezium cluster. However, this would not entirely explain

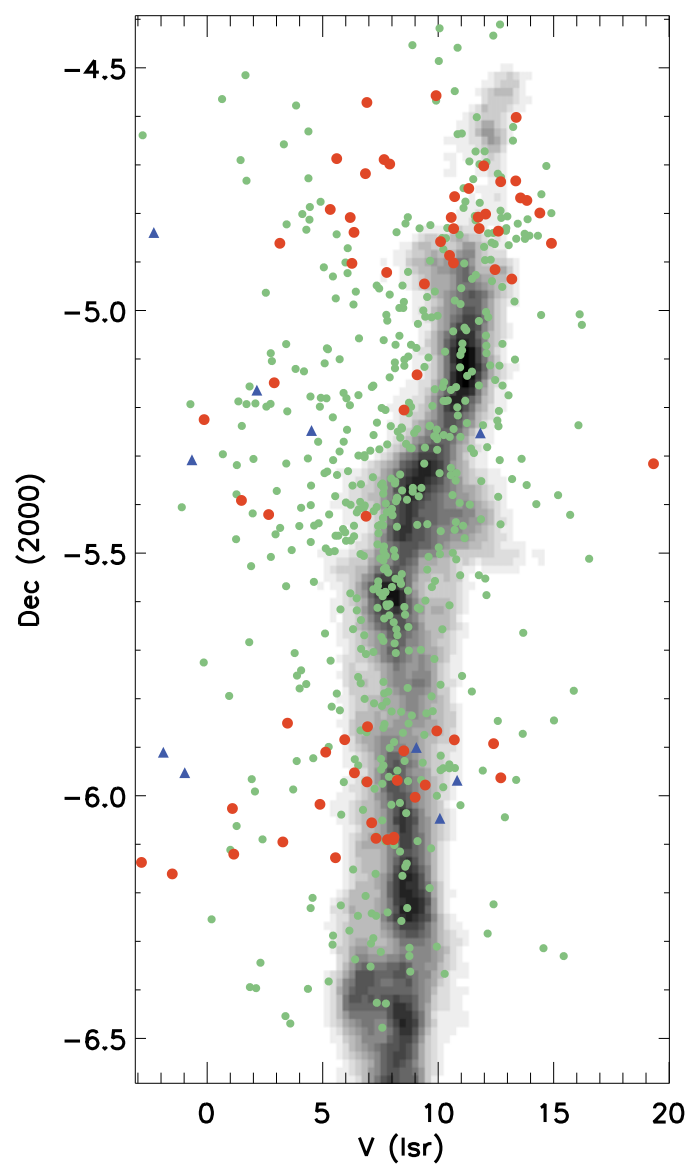

Figure 12. Position-velocity diagram for the ONC region, summed in right ascention. ${ }^{13} \mathrm{CO}$ map from Bally et al. (1987) is plotted in the background in grayscale. All the overplotted data points are non-binary sources that were observed in at least three epochs. Orange circles show sources where $\mathrm{Li}$ i has been detected, blue triangles show those that have been surveyed for the presence of $\mathrm{Li}$ I but did not produce detections. Green dots are all of the remaining sources for which no $\mathrm{Li}$ I information is available.

the presence of the blueshifted stellar population relative to the gas, and the location of these blueshifted stars is not correlated with either being on or off of the filament. More precise distances and proper motions, which could in part be obtained by the ongoing Gaia mission, are needed to confirm or deny the infall of the OMC $2 / 3$.

\section{2. $N G C 2264$}

While there is little substantial difference between the velocities for the NGC 2264 region quoted in T15 and this paper, some improvements could be made to the previous analysis of the velocity structure for the region through better filtering of the spectroscopic binaries. Similar to the case of the ONC, we restrict our analysis only to those sources that were detected in at least three epochs and show no RV variability. The position-velocity diagram for the stars is compared to that for gas from Ridge et al. (2003) in Figures 14 and 15.

We impose a constraint on sources to have an R.A. of $100.05 \geqslant \alpha \geqslant 100.4$ in order to only trace those objects that are spatially correlated with ${ }^{13} \mathrm{CO}$ gas to limit contamination from foreground or background sources. As a result, stars that are located north of $\delta \sim 9^{\circ} .55$ (i.e., Spokes Cluster and S Mon) have an agreement in RV with that of the gas that is significantly better than what was presented before by T15. 

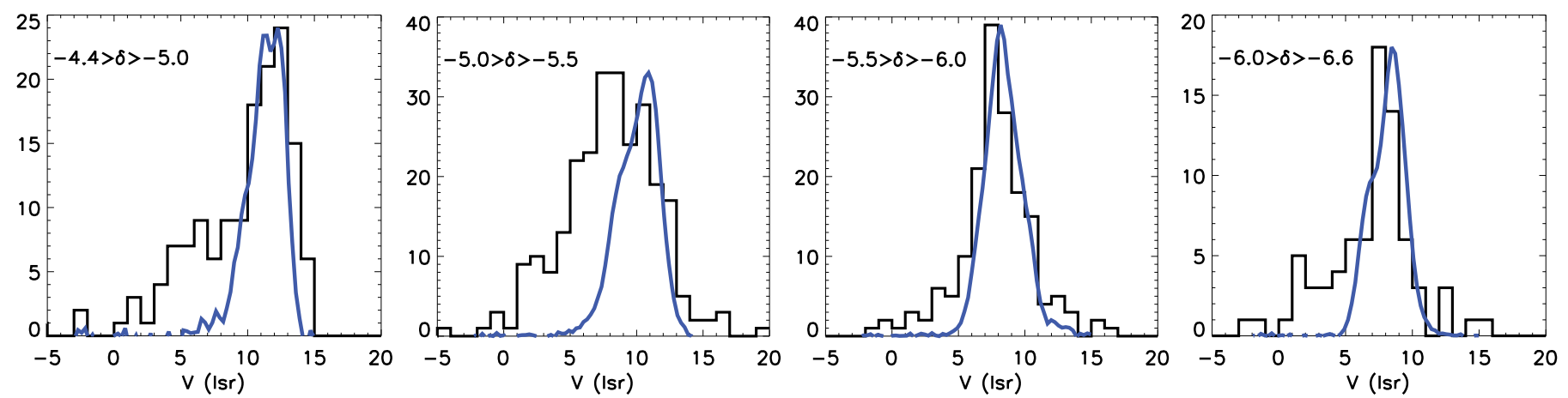

Figure 13. In black: distribution of the velocities of stars plotted in Figure 12 at four declination cuts. In blue: summed distribution of ${ }^{13} \mathrm{CO}$ at those declinations scaled to the peak of the histogram.

Table 4

Sources Surveyed for the Presence of Li I

\begin{tabular}{|c|c|c|c|c|c|c|c|c|}
\hline $\begin{array}{l}\mathrm{RV} \\
\#^{\mathrm{a}}\end{array}$ & $\begin{array}{l}\text { R.A. } \\
\text { (J2000) }\end{array}$ & $\begin{array}{l}\text { Decl. } \\
\text { (J2000) }\end{array}$ & $\begin{array}{c}v_{\mathrm{Li}}^{\mathrm{b}} \\
\left(\mathrm{km} \mathrm{s}^{-1}\right)\end{array}$ & $\begin{array}{c}\sigma_{\mathrm{Li}} \\
\left(\mathrm{km} \mathrm{s}^{-1}\right)\end{array}$ & $R_{\mathrm{Li}}$ & $\begin{array}{l}\lambda_{\mathrm{Li}}{ }^{\mathrm{c}} \\
(\stackrel{\mathrm{A}}{)})\end{array}$ & $\begin{array}{c}W_{\mathrm{Li}}^{\mathrm{d}} \\
(\AA)\end{array}$ & $\begin{array}{l}\text { Temp } \\
(\mathrm{K})\end{array}$ \\
\hline 113 & $05: 34: 15.45$ & $-06: 06: 55.1$ & 49.51 & 0.72 & 10.58 & $\ldots$ & $\ldots$ & 3750 \\
\hline 120 & 05:34:17.78 & $-05: 55: 43.1$ & 4.52 & 0.99 & 7.69 & $\ldots$ & $\ldots$ & 6000 \\
\hline 160 & $05: 34: 27.34$ & $-05: 24: 22.2$ & 26.02 & 2.26 & 9.18 & 6708.46 & 0.47 & 4000 \\
\hline 164 & 05:34:28.22 & $-05: 59: 09.0$ & 33.56 & 0.82 & 10.78 & 6708.62 & 0.10 & 6250 \\
\hline
\end{tabular}

Notes.

a Sources with RV \# greater than 2057 have $R<6$ for all detections, and thus they are not included in the Tables 2 and 3.

${ }^{\mathrm{b}}$ Velocity and other properties were measured only from $\mathrm{Li}$ I data.

${ }^{\mathrm{c}}$ Typical uncertainty in $\lambda_{\mathrm{Li}}$ is $0.01 \AA$.

d Typical uncertainty in $W_{\mathrm{Li}}$ is $0.01 \AA$.

(This table is available in its entirety in machine-readable form.)

Table 5

Orbital Parameters for Double-lined Binaries

\begin{tabular}{|c|c|c|}
\hline Parameter & RV 1768 & RV 1659 \\
\hline \multicolumn{3}{|c|}{ Adjusted Quantities } \\
\hline$P$ (day) & $4.7878 \pm 0.0002$ & $15.3182 \pm 0.0007$ \\
\hline$T_{p}(\mathrm{HJD})$ & $2454289.50 \pm 0.06$ & $2454294.07 \pm 0.05$ \\
\hline$e$ & $0.55 \pm 0.05$ & $0.00 \pm 0.02$ \\
\hline$\omega(\operatorname{deg})$ & $139.68 \pm 3.16$ & $0.00 \pm 0.60$ \\
\hline$\gamma\left(\mathrm{km} \mathrm{s}^{-1}\right)$ & $16.05 \pm 0.54$ & $22.83 \pm 0.46$ \\
\hline$K_{1}\left(\mathrm{~km} \mathrm{~s}^{-1}\right)$ & $59.34 \pm 6.34$ & $41.56 \pm 1.07$ \\
\hline$K_{2}\left(\mathrm{~km} \mathrm{~s}^{-1}\right)$ & $60.83 \pm 6.32$ & $52.20 \pm 1.05$ \\
\hline \multicolumn{3}{|c|}{ Derived Quantities } \\
\hline$M_{1} \sin ^{3} i\left(M_{\odot}\right)$ & $0.254 \pm 0.066$ & $0.73 \pm 0.04$ \\
\hline$M_{2} \sin ^{3} i\left(M_{\odot}\right)$ & $0.248 \pm 0.065$ & $0.58 \pm 0.03$ \\
\hline$q=M_{2} / M_{1}$ & $0.98 \pm 0.15$ & $0.80 \pm 0.03$ \\
\hline$a_{1} \sin i\left(10^{6} \mathrm{~km}\right)$ & $3.26 \pm 0.37$ & $8.75 \pm 0.23$ \\
\hline$a_{2} \sin i\left(10^{6} \mathrm{~km}\right)$ & $3.35 \pm 0.37$ & $11.00 \pm 0.22$ \\
\hline$a \sin i\left(10^{6} \mathrm{~km}\right)$ & $6.61 \pm 0.52$ & $19.75 \pm 0.32$ \\
\hline \multicolumn{3}{|c|}{ Other Quantities } \\
\hline$\chi^{2}$ & 8.38 & 13.43 \\
\hline$N_{\text {obs }}$ (primary) & 10 & 9 \\
\hline$N_{\text {obs }}$ (secondary) & 8 & 8 \\
\hline Time span (days) & 2308.7 & 2308.6 \\
\hline $\mathrm{rms}_{1}\left(\mathrm{~km} \mathrm{~s}^{-1}\right)$ & 1.62 & 1.22 \\
\hline $\mathrm{rms}_{2}\left(\mathrm{~km} \mathrm{~s}^{-1}\right)$ & 1.42 & 2.10 \\
\hline$T_{\text {ave }}(\mathrm{K})$ & 4021 & 4203 \\
\hline
\end{tabular}

This is partially due to the stricter spatial constraints than were originally imposed. No objects exhibit a significant blueshift in RV relative to the gas, with the slight exception of the southernmost declinations in the Spokes Cluster.

However, the entirety of the stellar population found toward the Cone Nebula does show a significant blueshift that is not dissimilar to what is found toward the Trapezium and OMC 2/ 3 regions in the ONC. However, unlike Trapezium where the dispersion velocity of the stars is wide enough to also correlate with the gas, the stars toward the Cone Nebula appear to be decoupled from the gas. The reason for this is not entirely clear.

In addition to these regions, there appears to be a small cluster of stars centered at $\alpha \sim 100^{\circ} .45, \delta \sim 9^{\circ} .7$ with a diameter of $\sim 0^{\circ} .1$ (Figure 14). It was previously identified but not discussed in T15. The members of this cluster appear to have a systematic $\mathrm{RV}_{\mathrm{lsr}} \sim 2 \mathrm{~km} \mathrm{~s}^{-1}$, which is somewhat distinguishable from the main cluster. It is possible that it is a an older cluster that has managed to clear away all of its gas.

Since this cluster does not appear to be dynamically relaxed, as it exhibits a significant distinct substructure, a better determination of its age and further modeling will be needed to determine the degree of interaction between these subclusters. This could shed light on the dominant method of cluster formation, such as whether or not it is undergoing cold collapse (Scally \& Clarke 2002).

\section{CONCLUSIONS}

In this paper, we continue the efforts started in T09 and T15 toward characterizing the stellar RVs of the two closest 
Table 6

Orbital Parameters for Single-lined Binaries

\begin{tabular}{|c|c|c|c|c|c|c|}
\hline Parameter & RV 1166 & RV 1372 & RV 1496 & RV 1550 & RV 1660 & RV 1753 \\
\hline \multicolumn{7}{|c|}{ Adjusted Quantities } \\
\hline$P$ (day) & $105.82 \pm 0.27$ & $315.41 \pm 5.79$ & $588.93 \pm 9.47$ & $72.83 \pm 0.03$ & $622.00 \pm 26.32$ & $12.93 \pm 0.01$ \\
\hline$T_{p}(\mathrm{HJD})$ & $2452620.5 \pm 29.3$ & $2454382.4 \pm 5.5$ & $2454814.5 \pm 22.1$ & $2454329.0 \pm 1.1$ & $2454585.1 \pm 31.4$ & $2454299.3 \pm 0.6$ \\
\hline$e$ & $0.00 \pm 0.07$ & $0.65 \pm 0.12$ & $0.44 \pm 0.07$ & $0.32 \pm 0.02$ & $0.56 \pm 0.08$ & $0.170 \pm 0.03$ \\
\hline$\omega(\operatorname{deg})$ & $224.02 \pm 99.11$ & $178.80 \pm 5.31$ & $8.61 \pm 11.29$ & $266.43 \pm 7.12$ & $135.59 \pm 11.99$ & $194.66 \pm 19.74$ \\
\hline$\gamma\left(\mathrm{km} \mathrm{s}^{-1}\right)$ & $15.93 \pm 0.25$ & $-29.97 \pm 0.78$ & $28.05 \pm 0.30$ & $20.80 \pm 0.26$ & $23.08 \pm 0.39$ & $17.97 \pm 1.35$ \\
\hline$K_{1}\left(\mathrm{~km} \mathrm{~s}^{-1}\right)$ & $4.24 \pm 0.46$ & $20.00 \pm 7.73$ & $3.46 \pm 0.87$ & $16.91 \pm 0.39$ & $5.86 \pm 0.51$ & $16.11 \pm 1.61$ \\
\hline \multicolumn{7}{|c|}{ Derived Quantities } \\
\hline$a_{1} \sin i\left(10^{6} \mathrm{~km}\right)$ & $6.17 \pm 0.67$ & $65.91 \pm 27.09$ & $25.09 \pm 6.38$ & $16.06 \pm 0.39$ & $41.36 \pm 4.93$ & $2.82 \pm 0.28$ \\
\hline$f\left(m_{1}, m_{2}\right)\left(M_{\odot}\right)$ & $0.0008 \pm 0.0003$ & $0.11 \pm 0.15$ & $0.0018 \pm 0.0014$ & $0.031 \pm 0.003$ & $0.007 \pm 0.003$ & $0.005 \pm 0.002$ \\
\hline \multicolumn{7}{|c|}{ Other Quantities } \\
\hline$\chi^{2}$ & 1.31 & 2.61 & 1.74 & 1.76 & 2.61 & 2.61 \\
\hline$N_{\text {obs }}$ (primary) & 10 & 11 & 10 & 11 & 11 & 11 \\
\hline Time span (days) & 903.6 & 2308.7 & 2327.7 & 2325.7 & 2325.6 & 2327.7 \\
\hline $\mathrm{rms}_{1}\left(\mathrm{~km} \mathrm{~s}^{-1}\right)$ & 0.17 & 0.53 & 0.19 & 0.25 & 0.55 & 0.28 \\
\hline$T_{\text {ave }}(\mathrm{K})$ & 6274 & 4267 & 5577 & 4361 & 4256 & 5301 \\
\hline
\end{tabular}

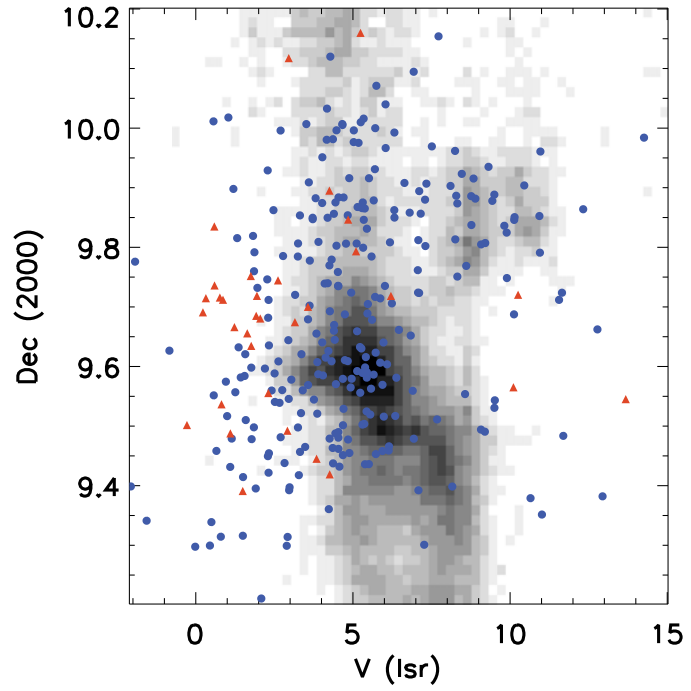

Figure 14. Position-velocity diagram for the NGC 2264 region, summed in right ascention. ${ }^{13} \mathrm{CO}$ map from Ridge et al. (2003) is plotted in the background in grayscale. All the overplotted data points are non-binary sources that were observed in at least three epochs. Blue dots have R.A. range between 100.05 and $100^{\circ} .4$, orange triangles range between $100^{\circ} .4$ and $100^{\circ} .5$ to show a subcluster centered at $\alpha \sim 100{ }^{\circ} 45, \delta \sim 99^{\circ} .7$.

massive star-forming regions, the ONC and NGC 2264. Using multi-epoch observations, we search for sources that exhibit a significant change in RV that could be attributed to the presence of a binary. We identify a total of 130 multiple system between the two regions. For 8 of the sources located in NGC 2264 we produce detailed orbital fits, and for two of these sources we can determine a mass ratio between the primary and the secondary.

The multiplicity fraction that we observe is $5.8 \pm 1.1 \%$ for the ONC and $5.3 \pm 1.2 \%$ for NGC 2264. If these systems were consistent with what is observed in nearby $G$ dwarfs, then, considering the uncertainties of individual measurements and
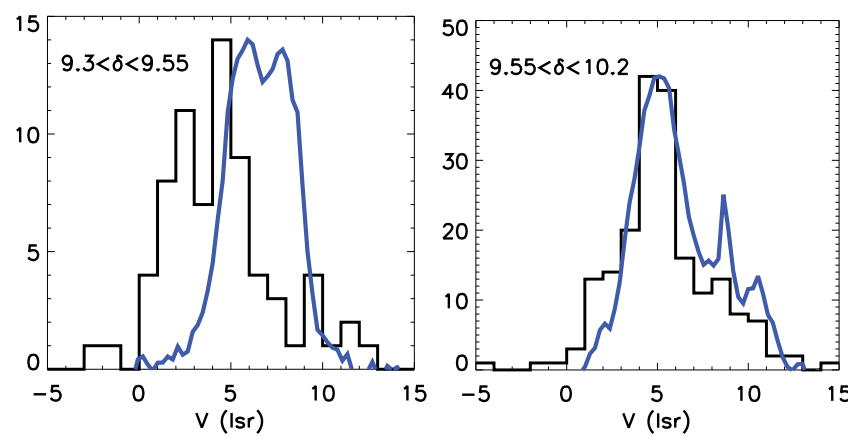

Figure 15. Black: distribution of the velocities of stars plotted in Figure 14 at two declination cuts. A R.A range between 100.05 and $100{ }^{\circ} .4$ has been imposed to minimize contamination from sources outside of the main cluster. Blue: summed distribution of ${ }^{13} \mathrm{CO}$ at those declinations scaled to the peak of the histogram.

allowing primaries of $0.7 M_{\odot}$, we would expect to observe MFs of $4.5 \pm 0.9 \%$ and $5.8 \pm 1.2 \%$, respectively, for these two clusters. Both NGC 2264 and ONC have a distribution of multiple stars that is largely consistent with what is observed in the field within the same parameter space. However, a study of the wide binaries in NGC 2264 would be needed to conclusively compare the MF of these two regions.

In addition to analyzing multiplicity, we reexamined the stellar RV distribution relative to that of gas for both of these clusters to find significantly better agreement between the two than has been previously reported, as both the peak of the distribution and the velocity dispersion of stars and gas are extremely similar in many regions of these clusters. The presence of the blueshifted young stars is reduced significantly in the cleaned sample, but they are not entirely absent. Some of these sources could be explained by the specifics of the star formation processes in these regions, for example, by stellar feedback pushing the gas away or by the presence of a separate foreground cluster. 
J.J.T. is currently supported by grant 639.041.439 from the Netherlands Organization for Scientific Research (NWO).

\section{REFERENCES}

Adams, F. C. 2010, ARA\&A, 48, 47

Alves, J., \& Bouy, H. 2012, A\&A, 547, A97

Bally, J., Langer, W. D., Stark, A. A., \& Wilson, R. W. 1987, ApJL, 312, L45 Baraffe, I., Homeier, D., Allard, F., \& Chabrier, G. 2015, A\&A, 577, A42

Baxter, E. J., Covey, K. R., Muench, A. A., et al. 2009, AJ, 138, 963

Bernstein, R., Shectman, S. A., Gunnels, S. M., Mochnacki, S., \& Athey, A. E. 2003, Proc. SPIE, 4841, 1694

Bouy, H., Alves, J., Bertin, E., Sarro, L. M., \& Barrado, D. 2014, A\&A, 564, A29

Briceno, C., Hartmann, L. W., Stauffer, J. R., et al. 1997, AJ, 113, 740

Cutri, R. M., Skrutskie, M. F., van Dyk, S., et al. 2003, yCat, 2246, 0

Da Rio, N., Tan, J. C., Covey, K. R., et al. 2015, arXiv:1511.04147

Donati, J.-F., Gregory, S. G., Alencar, S. H. P., et al. 2013, MNRAS, 436, 881

Donati, J.-F., Hébrard, E., Hussain, G., et al. 2014, MNRAS, 444, 3220

Donati, J.-F., Hébrard, E., Hussain, G. A. J., et al. 2015, MNRAS, 453, 3706

Duchêne, G., \& Kraus, A. 2013, ARA\&A, 51, 269

Duquennoy, A., \& Mayor, M. 1991, A\&A, 248, 485

Elmegreen, B. G. 2007, ApJ, 668, 1064

Fischer, D. A., \& Marcy, G. W. 1992, ApJ, 396, 178

Fűrész, G., Hartmann, L. W., Megeath, S. T., Szentgyorgyi, A. H., \& Hamden, E. T. 2008, ApJ, 676, 1109

Fưrész, G., Hartmann, L. W., Szentgyorgyi, A. H., et al. 2006, ApJ, 648, 1090

Hartmann, L., \& Burkert, A. 2007, ApJ, 654, 988

Hennebelle, P. 2012, A\&A, 545, A147

Hillenbrand, L., Isaacson, H., Marcy, G., et al. 2015, in Proc. 18th Cambridge Workshop on Cool Stars, Stellar Systems, and the Sun 18, ed. G. T. van Belle, \& H. C. Harris, 759

Hillenbrand, L. A. 1997, AJ, 113, 1733

Iglesias-Marzoa, R., López-Morales, M., \& Jesús Arévalo Morales, M. 2015, PASP, 127, 567
Karnath, N., Prato, L., Wasserman, L. H., et al. 2013, AJ, 146, 149

Kerr, F. J., \& Lynden-Bell, D. 1986, MNRAS, 221, 1023

Köhler, R., Petr-Gotzens, M. G., McCaughrean, M. J., et al. 2006, A\&A, 458,461

Kounkel, M. A., Megeath, S. T., Poteet, C., Fischer, W. J., \& Hartmann, L. 2016, ApJ, in press

Kouwenhoven, M. B. N., Brown, A. G. A., Portegies Zwart, S. F., \& Kaper, L. 2007, A\&A, 474, 77

Kurtz, M. J., \& Mink, D. J. 1998, PASP, 110, 934

Kuznetsova, A., Hartmann, L., \& Ballesteros-Paredes, J. 2015, ApJ, 815, 27

Mateo, M., Bailey, J. I., Crane, J., et al. 2012, Proc. SPIE, 8446, 4

Menten, K. M., Reid, M. J., Forbrich, J., \& Brunthaler, A. 2007, A\&A, 474,515

Munari, U., Sordo, R., Castelli, F., \& Zwitter, T. 2005, A\&A, 442, 1127

Proszkow, E.-M., Adams, F. C., Hartmann, L. W., \& Tobin, J. J. 2009, ApJ, 697, 1020

Raghavan, D., McAlister, H. A., Henry, T. J., et al. 2010, ApJS, 190, 1

Reipurth, B., Guimarães, M. M., Connelley, M. S., \& Bally, J. 2007, AJ, 134,2272

Ridge, N. A., Wilson, T. L., Megeath, S. T., Allen, L. E., \& Myers, P. C. 2003, AJ, 126, 286

Rizzuto, A. C., Ireland, M. J., Robertson, J. G., et al. 2013, MNRAS, 436, 1694

Sandstrom, K. M., Peek, J. E. G., Bower, G. C., Bolatto, A. D., \& Plambeck, R. L. 2007, ApJ, 667, 1161

Scally, A., \& Clarke, C. 2002, MNRAS, 334, 156

Sicilia-Aguilar, A., Hartmann, L. W., Fürész, G., et al. 2006, AJ, 132, 2135

Sung, H., Bessell, M. S., \& Lee, S.-W. 1997, AJ, 114, 2644

Szentgyorgyi, A. H., Cheimets, P., Eng, R., et al. 1998, Proc. SPIE, 3355, 242

Tan, J. C., Krumholz, M. R., \& McKee, C. F. 2006, ApJL, 641, L121

Tobin, J. J., Hartmann, L., Furesz, G., Hsu, W.-H., \& Mateo, M. 2015, AJ, 149,119

Tobin, J. J., Hartmann, L., Furesz, G., Mateo, M., \& Megeath, S. T. 2009, ApJ, 697, 1103

Tonry, J., \& Davis, M. 1979, AJ, 84, 1511

Walker, M. G., Mateo, M., Olszewski, E. W., et al. 2007, ApJS, 171, 389 